\title{
Papillary thyroid cancer presenting as a giant mediastinal cyst
}

\author{
John Young ${ }^{1}$, Anne Granfield ${ }^{1}$, Peter J Mazzaglia ${ }^{1}$ and Ikenna Okereke ${ }^{2 *}$ \\ ${ }^{1}$ Department of Surgery, Warren Alpert Medical School of Brown University, Providence, RI, USA \\ ${ }^{2}$ Division of Cardiothoracic Surgery, University of Texas Medical Branch, Galveston, TX, USA
}

\section{Case report}

A 75 year old male presented to our institution for evaluation of shortness of breath and a 20 pound weight loss over one year. He had no palpable neck masses and denied dysphagia. Computed tomography scan of the chest revealed a $16.5 \times 7.8 \times 9.6$ centimeter, predominantly cystic mass extending from just above the cervico-thoracic junction inferiorly into the mediastinum and to the right of the midline (Figure 1). The mass caused significant leftward deviation of the trachea and larynx. There was a solid component with calcifications in the superior and medial aspect of the lesion. The patient underwent a right thoracoscopic biopsy of the cyst wall and evacuation of intracystic contents. During the procedure 700 milliliters of serous fluid were drained from the mediastinal cyst. There was fibrinous material noted within the cyst and the anterolateral wall was removed to marsupialize the cyst.

Intraoperative frozen section was negative for malignancy. Pathologic analysis of the cyst wall showed papillary structures with hyperplastic epithelium and atypical pleomorphic nuclei, many with nuclear pseudo inclusions, and subsets with abundant eosinophilic cytoplasm or hobnail appearance. Immunohistochemistry was positive for thyroglobulin, CK7, CK19, and TTF-1. These findings were consistent with papillary thyroid cancer.

Endocrine surgery consultation was obtained. A neck ultrasound revealed the right thyroid lobe to be replaced by a hypoechoic irregular mass with multiple calcifications measuring $3.6 \times 3.0$ centimeters, with adjacent abnormal appearing level IV lymph nodes. As the patient had developed hoarseness, a flexible laryngoscopy was performed which demonstrated right vocal cord paralysis.

One month later, the patient was brought back to the operating room. A nerve monitoring endo-tracheal tube was employed. A total

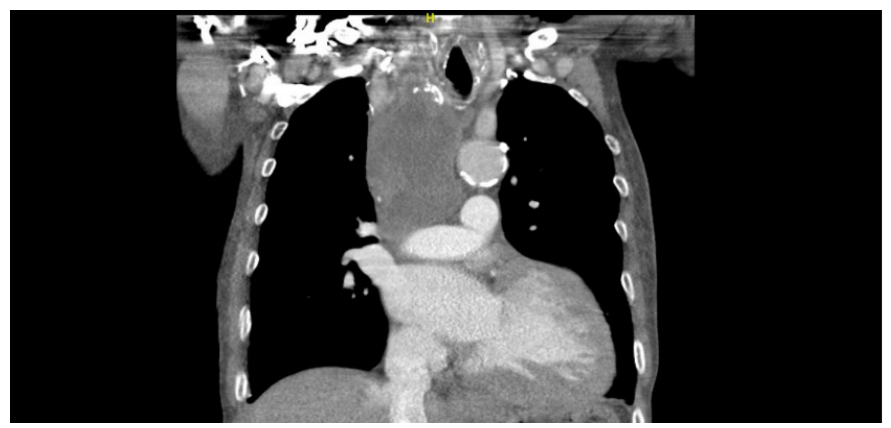

Figure 1. Coronal CT of mass. thyroidectomy, sternotomy and excision of the mediastinal mass were performed. The mass was adherent to the right pleura but did not invade the lung or the pulmonary vasculature. No pulmonary resection was required, as the mass was easily separated from the lungs. The mass abutted the superior vena cava but did not invade it and was able to be peeled off it without difficulty. No pericardial resection was necessary. The right recurrent laryngeal nerve was involved with the mass and was resected en bloc. The ansa cervicalis was mobilized and anastomosed to the distal portion of the right recurrent laryngeal nerve. Due to the extensive nature of the dissection, it was necessary to autotransplant the right parathyroid glands. The entire mass was ultimately removed with negative margins.

The patient was extubated on post-operative day 1 and discharged on post-operative day 5 in good condition. His hoarseness began to resolve immediately post-operatively and was almost completely eliminated by the time of discharge. Final surgical pathology revealed a 16.5 centimeter cystic mass involving the right lobe of the thyroid and mediastinum with scattered foci of papillary thyroid cancer. The largest contiguous focus of carcinoma was 6 millimeters (Figure 2). All surgical margins were negative. Interestingly, 1 of 11 lymph nodes demonstrated an incidental 5 millimeter focus of involvement of both Grade II follicular lymphoma and diffuse large B-cell lymphoma (DLBCL). There was no evidence of metastatic thyroid cancer to the lymph nodes.

\section{Comment}

While mediastinal tumors related to thyroid cancer from an ectopic thyroid or direct goiter extension have been reported, a large cystic mediastinal lesion associated with an unrecognized thyroid cancer is rare $[1,2]$. These patients present primarily with compressive symptoms such as cough, dyspnea, or dysphagia [3-5]. The surgical pathology of the thyroidectomy specimen often yields a tumor size of less than 1 cubic centimeter in these cystic lesions, contributing to the difficulty in diagnosing malignancy using percutaneous biopsy [1].

Our patient presented with shortness of breath and weight loss

Correspondence to: Ikenna Okereke, M.D., FACS, Associate Professor of Surgery, Chief, Thoracic Surgery, University of Texas Medical Branch, 301 University Blvd, Galveston, TX 77555, Tel: 409-772-1203, Fax: 409-772-1421; E-mail: ikokerek@UTMB.edu

Key words: mediastinal tumor, pathology (thyroid)

Received: February 14, 2016; Accepted: March 18, 2016; Published: March 21, 2016 


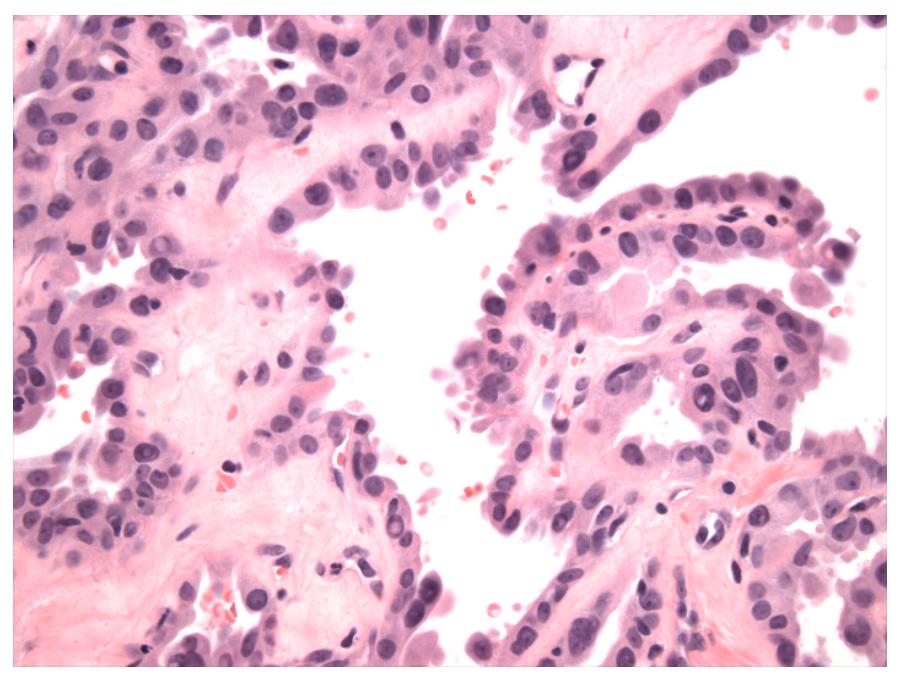

Figure 2. High power $\mathrm{H} \& \mathrm{E}$.

with no palpable neck masses and a CT scan showing a mediastinal cyst. Given the rare nature of thyroid malignancy presenting as a mediastinal cyst, the diagnosis of papillary thyroid cancer was low on the differential diagnosis. The lack of any tumor focus larger than 6 millimeters in our specimen also would have made diagnosing thyroid cancer prior to the initial operation challenging. However, once the diagnosis of papillary thyroid cancer was made, an aggressive surgical resection was undertaken. Given the size of the mass and adherence to mediastinal structures, a sternotomy was required and was successful in achieving negative margins. We elected to anastomose the ansa cervicalis to the recurrent laryngeal nerve in the hope that the patient would ultimately have some remaining tone in the vocal cord. We were pleasantly surprised that the patient had virtually no hoarseness by the time of discharge, but we feel that immediate functionality of the paralyzed vocal cord from our ansa cervicalis anastomosis is highly unlikely. It is possible instead that edema of the vocal cords caused by intubation during the second procedure helped to resolve the hoarseness. If the hoarseness returns we will perform a repeat laryngoscopy to assess vocal cord function, but the patient has had no recurrent symptoms to date.

While rare, thyroid cancer can present as a giant, cystic mediastinal mass. A high clinical suspicion should be maintained in the evaluation of these patients so appropriate preoperative evaluation and planning can be performed. When diagnosed, complete surgical resection should be performed if technically possible.

\section{References}

1. Bhamidipati CM, Mukhopadhyay S, Feliu C, Patton B, Dexter E (2009) Cystic metastases of papillary thyroid carcinoma mimicking a primary mediastinal cyst. $J$ ThoracOncol4: 105-106. [Crossref]

2. Suemitsu R, Takeo S, Hamatake M, Yamamoto T, Furuya K, et al. (2010) Thyroid cancer with a cystic mediastinal tumor invading the right main bronchus. Ann ThoracSurg 89: 296-298. [Crossref]

3. Shah BC, Ravichand CS, Juluri S, Agarwal A, Pramesh CS, et al. (2007) Ectopic thyroid cancer. Ann ThoracCardiovascSurg 13: 122-124. [Crossref]

4. Ket S, Ozbudak O, Ozdemir T, Dertsiz L (2004) Acute respiratory failure and tracheal obstruction in patients with posterior giant mediastinal (intrathoracic) goiter. Interact CardiovascThoracSurg 3: 174-175. [Crossref]

5. Katlic MR, Wang CA, Grillo HC (1985) Substernal goiter. Ann ThoracSurg39: 391399. [Crossref]

Copyright: (C2016 Young J. This is an open-access article distributed under the terms of the Creative Commons Attribution License, which permits unrestricted use, distribution, and reproduction in any medium, provided the original author and source are credited. 